\title{
Long-lived Charge-Transfer States in 9-Aryl-Acridinium Ions; A Critical Reinvestigation
}

\author{
Jan W. Verhoeven, ${ }^{1, \dagger}$ Hendrik J. van Ramesdonk, ${ }^{1}$ Hong Zhang, ${ }^{1}$ \\ Michiel M. Groeneveld, ${ }^{1}$ Andrew C. Benniston, ${ }^{2}$ and Anthony Harriman ${ }^{2}$ \\ ${ }^{1}$ Department of Chemistry, HIMS, University of Amsterdam, Nieuwe Achtergracht 129, \\ 1018 WS Amsterdam, Netherlands \\ ${ }^{2}$ Molecular Photonics Laboratory, School of Natural Sciences - Chemistry, Bedson Building, \\ University of Newcastle, Newcastle upon Tyne NE1 7RU, United Kingdom
}

\begin{abstract}
In the recent literature a simple 9-aryl-acridinium ion was claimed to undergo an intramolecular, photoinduced charge shift to produce an extremely long-lived and very high energy charge-transfer state. The possible consequences of this observation are discussed and the tenability of the claims made is investigated via time resolved spectroscopy of a closely related system with spectroscopic characteristics allowing more solid identification of the actual photophysical events taking place. From the results obtained it appears likely that the long-lived species observed earlier in solution cannot be charge transfer in nature but must instead be identified as the lowest triplet state of the acridinium chromophore.
\end{abstract}

\section{INTRODUCTION}

Storage of light energy in a long-lived molecular chargetransfer state is a much pursued goal. Such molecular systems would be of importance for energy conversion analogous to natural photosynthesis and might also be applied in the construction of molecular photoelectromechanical and photo-electric devices.

Crucial in photosynthetic energy conversion is the occurrence of a number of relatively fast short-range electron-transfer steps leading to a large overall charge separation distance which prevents the loss of the photoinduced redox potential as a result of rapid back electron transfer. Mimicking this has been achieved after decades of scientific effort but evidently requires quite complex and large multi-chromophoric systems ranging from triads to pentads [1-8].

Alternatively, attempts have been made to design simple and small dyad systems ("compact dyads") in such a way that not only photoinduced charge separation occurs rapidly and quantitatively but, at the same time, charge recombination is somehow impeded. The most successful of such systems seem to be those in which "spin control" is applied by populating a chargetransfer state which is of different spin multiplicity (e.g. a triplet) than the ground state (usually a singlet) whereby charge recombination becomes a spin forbidden process [9-12].

While all earlier results thus indicated that creation of a long-lived charge-transfer (CT) state is a complex issue, a very simple and compact dyad was recently reported in which a long-lived CT state was claimed to be populated without the need for long distance

\footnotetext{
†E-mail: jwv@science.uva.nl
}

charge migration nor for spin restrictions [13]. The longevity was attributed to a high thermal barrier for decay of the CT state. This barrier was assigned to a pronounced and unprecedented inverted region effect. Below we will discuss those claims and present an alternative explanation that puts serious doubts on their validity.

\section{LONG LIVED CHARGE MIGRATION IN A SIMPLE ARYL-ACRIDINIUM DYAD?}

An amazing example of a long-lived CT state in a very simple and compact dyad was recently reported in the form of the 9-mesityl-10-methylacridinium ion 1 (see Figure 1) [13]. This system contains a directly linked donor/acceptor pair comprising a mesitylene moiety as a relatively weak electron donor and an acridinium ion as a rather strong electron acceptor that can be excited with visible light. It was reported that excitation of the acridinium acceptor chromophore leads to population of an intramolecular CT state in which the mesityl moiety functions as donor. Although donor and acceptor moieties are almost perpendicular, it seems likely that sufficient electronic coupling will be available to make this charge-shift reaction fast. It therefore comes as no surprise that $\mathbf{1}$ was reported to undergo photoinduced charge shift in $\geq 98 \%$ quantum yield. Very surprising, however, is the fact that the CT state thus populated was claimed to have an extremely long lifetime ranging from many microseconds in solution to many hours and even years at lower temperatures in a solid matrix [13]. Based on these observations 1 was explicitly claimed to constitute a system that surpasses natural photosynthesis both regarding the amount of energy 
<smiles>Cc1cc(C)c(-c2c3ccccc3[n+]([Al-])c3ccccc23)c(C)c1</smiles><smiles></smiles>

Figure 1. Structure of the 9-mesityl-10-methylacridinium system 1 claimed to produce an extremely long-lived intramolecular CT state. The closely related system 2 is herein used as a model to check the identity of the long-lived state formed.

stored and the storage time. More recently these properties were also reported to allow $\mathbf{1}$ to act as a useful photoredox sensitizer $[14,15]$. The long lifetime of the CT state in 1 was attributed to a high barrier for back electron transfer under the inverted region conditions caused by the high energy $(2.37 \mathrm{eV})$ of the CT state in 1 [13]. This reasoning was especially based upon the strong temperature dependence of the very slow, first order decay kinetics of the CT state detected by EPR spectroscopy (no spectra shown) in a solid matrix between 203 and $243 \mathrm{~K}$. The identification of this CT state was, however, mainly based upon its transient absorption spectrum measured by flash photolysis in liquid solution at higher temperatures.

While we comment on the low temperature photoEPR experiments reported for $\mathbf{1}$ in a separate paper [16], in the present publication we concentrate specifically on the identification of its long-lived CT state by flash photolysis in solution.

We realized that the long lifetime of the CT state in 1 cannot be attributed to an eventual triplet character of this state because in $\mathbf{1}$ the lowest triplet state is not CT in nature but according to well documented literature data must be localized on the acridinium chromophore $\left(\mathrm{E}_{\mathrm{T}} \sim 2.0 \mathrm{eV}\right)[17,18]$. Furthermore we noted that in the visible region the transient absorption spectrum to be expected for the CT state of $\mathbf{1}$ is somewhat similar to that to be expected for the $T_{1}-T_{n}$ absorption of the acridinium chromophore [19]. This might make it difficult to discriminate between these species. We therefore now (re-)investigated the properties of 2 (see Figure 1), which is closely related to $\mathbf{1}$ but has the advantage that the transient absorption spectra of its CT state and its lowest triplet state are very different (see later). The only significant difference between $\mathbf{1}$ and $\mathbf{2}$ is that in the latter the CT state is at slightly lower energy (at ca. $2.14 \mathrm{eV}$ ) but-importantly in this context-still above the local acridinium triplet.

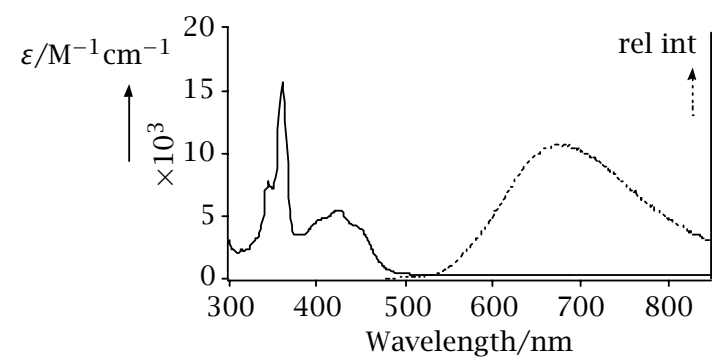

Figure 2. Electronic absorption and fluorescence of 2 in acetonitrile at room temperature.

2.1. Steady state absorption and fluorescence. The absorption and fluorescence spectra of 2 are shown in Figure 2. As expected the visible and near UV absorption of 2 is dominated by the acridinium chromophore with no clear indication for interaction with the naphthyl moiety orientated almost perpendicular to it. Such interaction is, however, very evident in the fluorescence spectrum.

Whereas the isolated acridinium ion is known to display a strong and structured green fluorescence (maximum around $495 \mathrm{~nm}$ ) [17, 20], this is fully quenched in 2 and substituted by a weak, broad and structureless emission in the far red region (maximum $675 \mathrm{~nm}$ in acetonitrile). This behaviour has been observed before and can safely be attributed to CTfluorescence, i.e. emissive back electron transfer from a ${ }^{1} \mathrm{CT}$ state populated by electron transfer from the naphthyl donor to the acridinium ion [21-23]. From the measured decay of the CT fluorescence, the lifetime of the CT state in 2 could be determined directly as $3.0 \mathrm{~ns}$. This value is in excellent agreement with earlier reports $[22,23]$.

2.2. Femtosecond transient absorption. In order to study the rise and decay pathways for the CT state in 2 in more detail laser flash photolysis measurements were performed with respectively subpicosecond and nanosecond temporal resolution.

Figure 3 shows the transient absorption spectra which evolve after femtosecond laser excitation $(430 \mathrm{~nm}$, fwhm $130 \mathrm{fs})$ of the acridinium chromophore in 2. Immediately after the laser pulse spectrum-1 is observed, with a maximum at $480 \mathrm{~nm}$ and a shoulder at $\sim 580 \mathrm{~nm}$, which may be attributed to $S_{1} \rightarrow S_{n}$ absorption of the acridinium chromophore. This is substituted on a subpicosecond time scale (risetime $\sim 0.4 \mathrm{ps}$, see Figure 4) by the spectrum of the CT state (spectrum-3), which remains stable over the observation period of the measurements ( $\leq 1 \mathrm{~ns}$ ) as expected from its lifetime of 3 ns determined from CT fluorescence (see above).

The spectrum of the CT state (i.e. spectrum-3 in Figure 3) displays features characteristic of both the neutral acridinyl radical [24], and the naphthalene 


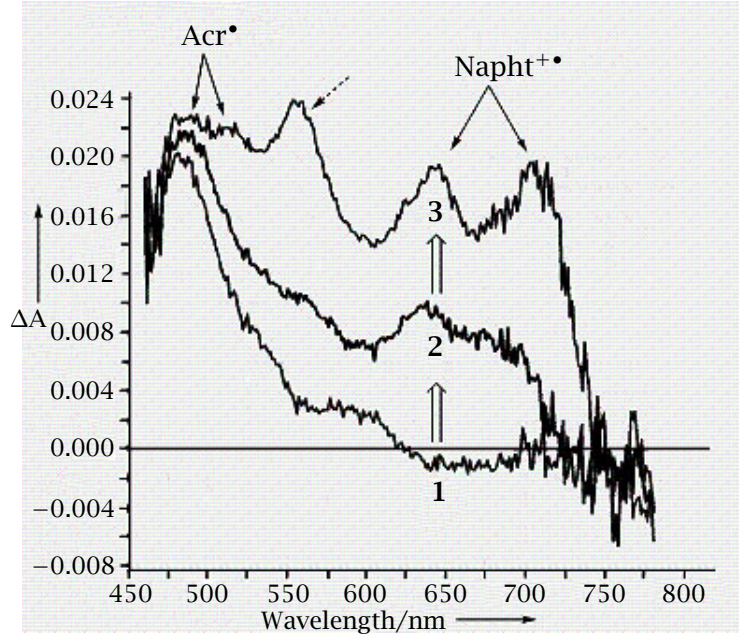

Figure 3. Transient absorption spectra of 2 observed at the delay times indicated in Figure 4 after femtosecond laser excitation at $430 \mathrm{~nm}$ ( $\mathrm{fwhm} 130 \mathrm{fs}$ ) in acetonitrile (pathlength $2 \mathrm{~mm}$ ). Spectral features attributable to the acridinyl radical (Acr ${ }^{\bullet}$ ) and the naphthalene radical cation (Napht ${ }^{+\bullet}$ ) moieties have been indicated by solid arrows. The dashed arrow indicates an extra band resulting from CT type interaction between these moieties (see text).

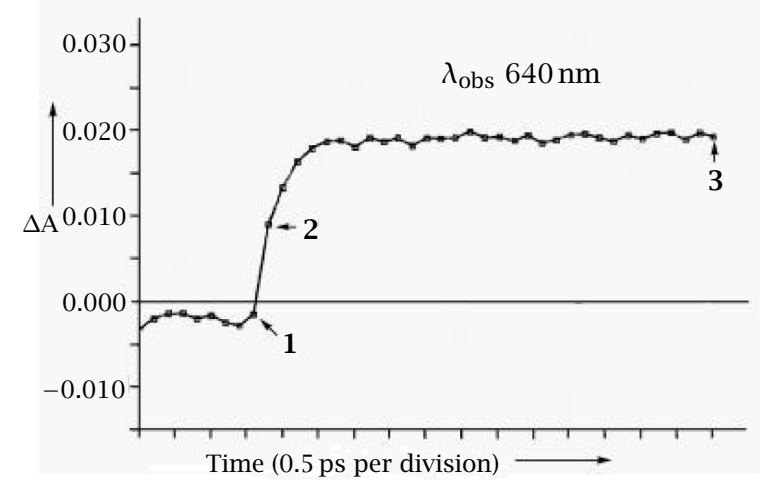

Figure 4. Rise curve for the CT state of 2 as detected at $640 \mathrm{~nm}$ by femtosecond transient absorption spectroscopy. Markers 1, 2, and 3 correspond to the spectra shown in Figure 3.

radical cation [25], as indicated in Figure 3. It is important to note that, in addition to these spectral features, the spectrum contains an extra and rather strong absorption around $560 \mathrm{~nm}$, which must be a result of interaction between the two constituent moieties. This observation, which seems to have been overlooked previously [22], may indicate that a reduction in the twist angle is part of the internal reorganization accompanying electron transfer. The resulting increased electronic interaction between the strongly electron- affinic naphthalene radical cation and the strongly electron-donating acridinyl radical moieties is expected to lead to rather intense transitions of the intramolecular CT type. Such behaviour is well known in the groundstate absorption spectra of 9-aryl acridinium ions when the aryl group has strong electron-donor properties [21]. Interestingly in the present case, excitation via the absorption due to the CT excited state of 2 will lead to a higher excited state, presumably localized on the naphthalene donor moiety as predicted from a simple MO diagram (see Figure 5).

\subsection{Nano-microsecond transient absorption.}

Within the time window of the femtosecond transient absorption measurements ( $\leq 1 \mathrm{~ns}$ ) the absorption spectrum of the CT state of 2 , shown in Figure 3, undergoes only minor changes. The only real change is a slight decay in intensity, as expected on the basis of the $3 \mathrm{~ns}$ lifetime found for the CT fluorescence. However, on a longer time scale as observed via nanosecond flash photolysis, this spectrum is replaced by the much simpler spectral profile shown in Figure 6.

This spectrum displays a single maximum in the visible region at $490 \mathrm{~nm}$ and a pronounced shoulder around $550 \mathrm{~nm}$. There is also a small but significant rise in absorption towards the red end of the spectral region investigated $(\leq 800 \mathrm{~nm})$. By comparison with literature data, this spectrum can safely be assigned to the local acridinium triplet state [18, 19]. As concluded before by others, this species arises from the CT state by intersystem crossing and back electron transfer [23]. This local triplet has a microsecond lifetime in deoxygenated solution at room temperature. Its triplet nature is further confirmed by the observation that addition of iodomethane, as a heavy atom containing co-solvent, up to $10 \%(\mathrm{v} / \mathrm{v})$ enhances the yield by a factor up to sevenfold (see Figure 7) and, at the same, time strongly quenches the CT fluorescence. These findings indicate that intersystem crossing to the local triplet state must occur with a quantum yield $\leq 14 \%$ in pure acetonitrile.

The observations described above for 2 fully agree with and augment earlier reports, especially regarding formation of the local acridinium $\mathrm{T}_{1}$ triplet as the only long-lived. However, these findings are in marked contrast with the claimed formation of a comparably longlived CT state in 1. This enigma is further pronounced by the observation that the transient absorption spectrum of the local acridinium triplet clearly observed for 2 (see Figures 6 and 7 ) is virtually identical to the spectrum attributed in the literature to the long-lived CT state of $\mathbf{1}$, even including the weak absorbance in the near infrared [13].

2.4. Reaction of the long-lived transient with anthracene. A property of the long-lived species derived from 1 and claimed to support its CT nature is that it reacts with anthracene to produce the 


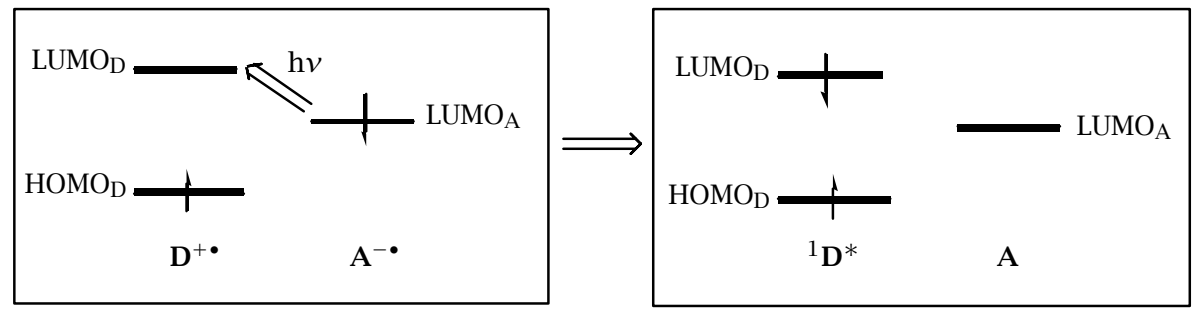

CT-state

Figure 5. Simplified MO diagram to show how excitation of the CT state of a D/A system can lead to the locally excited state of the donor.

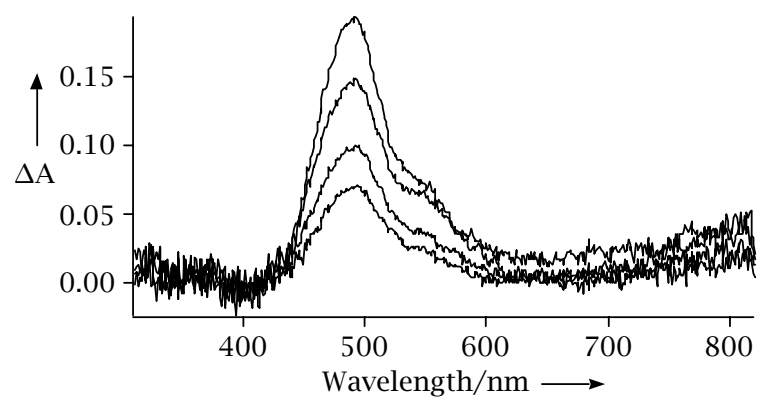

Figure 6. Long-lived transient absorption recorded for 2 in deoxygenated acetonitrile (conc. $0.5 \mathrm{mM}$, pathlength $1 \mathrm{~cm}$ ) at room temperature. Curves from top to bottom represent spectra observed with a delay time of $200 \mathrm{~ns}, 600 \mathrm{~ns}$, $1000 \mathrm{~ns}$ and $1400 \mathrm{~ns}$ respectively after laser excitation (450 nm, fwhm $2.7 \mathrm{~ns}, 2 \mathrm{~mJ}$ per pulse).

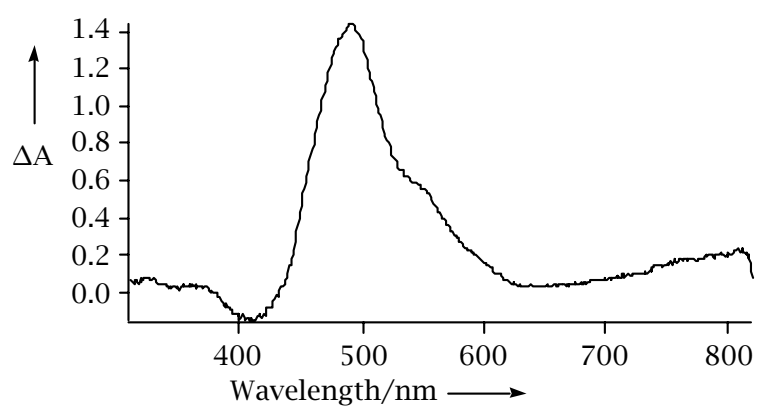

Figure 7. Transient absorption spectrum (delay $20 \mathrm{ns)} \mathrm{ob-}$ tained for 2 under identical conditions as in Figure 6 except for the addition of iodomethane $(10 \% \mathrm{v} / \mathrm{v})$. Note the ca. sevenfold increase in maximum absorbance.

anthracene radical cation [13]. However, this reaction was now also found to occur between anthracene and the local acridinium triplet derived from 2 . Thus (see Figure 8) anthracene (ca. $15 \mathrm{mM}$ ) is added to a solution of 2 in acetonitrile/iodomethane (10: $1 \mathrm{v} / \mathrm{v})$ and excited selectively via the acridinium chromophore with pulsed laser light $(450 \mathrm{~nm}, 2.7 \mathrm{~ns}$ fwhm, $2 \mathrm{~mJ}$ per pulse). Initially a spectrum very similar to that shown in
Figure 7 is observed which is dominated by that of the local acridinium triplet. This, however, rapidly evolves to a spectrum which can be attributed to a sum spectrum of the anthracene radical cation $\left(\mathrm{Anthr}^{+\bullet}\right.$ ) [25], and a spectrum typical of a neutral acridinyl radical $\left(\mathrm{Acr}^{\bullet}\right)$ [24]. In contrast to the rather narrow absorption maximum of the local acridinium triplet at $490 \mathrm{~nm}$ [19], acridinyl radicals show a much broader absorption around $500 \mathrm{~nm}$, which may even split up to a double maximum [24, 26].

These observations show that reaction (1) proceeds rapidly in solution and this implies that the acridinium triplet is a powerful oxidizing agent. The energetics of reaction (1) may be quantified by taking into account the ground state reduction potential of the acridinium ion $(-0.54$ V rel. to SCE in acetonitrile) [24], and the triplet energy of the acridinium ion (ca. $2 \mathrm{eV}$ ) [17], together with the one electron oxidation potential of anthracene $(+1.29 \mathrm{~V}$ rel. to SCE in acetonitrile) [27]. From these data reaction (1) is estimated to be exoergonic by ca. $0.17 \mathrm{eV}(3.91 \mathrm{kcal} / \mathrm{mol})$ and thus is expected to proceed to virtual completion under the circumstances employed. From the pseudo first order kinetics observed we estimate that the acridinium triplet of 2 enters into reaction (1) with a forward rate constant of $k \sim 1.5 \times 10^{9} \mathrm{M}^{-1} \cdot \mathrm{s}^{-1}$, which is quite comparable to that extracted from literature data for the analogous reaction of the long-lived state of $\mathbf{1}$ with anthracene $\left(k \sim 2 \times 10^{9} \mathrm{M}^{-1} \cdot \mathrm{s}^{-1}\right)$.

$$
{ }^{3}\left[\mathrm{Acr}^{+\bullet}\right]^{*}+\text { Anthr } \rightarrow \mathrm{Acr}^{\bullet}+\mathrm{Anthr}^{+\bullet}
$$

It should be noted that the strongly oxidizing properties of the acridinium triplet may also well explain other photoredox processes reported for $\mathbf{1}$ and (again) thought to require a long-lived CT state as the active species $[14,15]$.

\section{CONCLUDING REMARKS}

Based on the results obtained herein for 2 we tentatively conclude that, not only in 2 but in all likelihood also in $\mathbf{1}$, photoexcitation produces a CT state with 


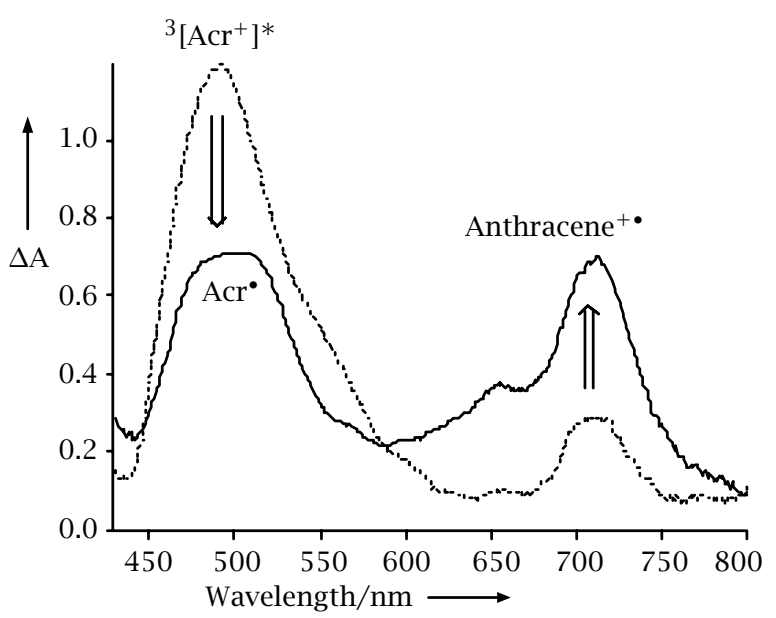

Figure 8. Reaction between anthracene and the local acridinium triplet state of $2={ }^{3}\left[\mathrm{Acr}^{+\cdot}\right]^{*}$, as induced by laser flash photolysis of 2 at $450 \mathrm{~nm}$ (2.7 $\mathrm{ns}$ fwhm, $2 \mathrm{~mJ}$ per pulse) in acetonitrile/iodomethane (10:1v/v). The dashed and solid curves represent spectra recorded respectively $8 \mathrm{~ns}$ and $30 \mathrm{~ns}$ after the laser pulse.

a lifetime in the nanosecond domain. Furthermore, the long-lived species observed for $\mathbf{1}$ by flash photolysis in solution is in fact the local triplet of its acridinium chromophore, as shown above for 2 .

Whether the very persistent EPR signals reported [13] for $\mathbf{1}$ after illumination in a solid matrix at low temperature are also attributable to such a local triplet state seems unlikely in view of what is known about the EPR behaviour of 2 under such conditions [23] as well as on the basis of photo-EPR results obtained by us with 1 [16]. We therefore feel the urgent need to reinvestigate the photophysical and photochemical properties of 1 in solution. Such re-investigations are underway at the time of writing and will be reported separately.

\section{EXPERIMENTAL}

4.1. Materials. Spectrograde acetonitrile was used as a solvent. All solutions used in time resolved spectroscopy were deoxygenated by bubbling with argon. The 9-(1-naphthyl)-10-methylacridinium chloride 2 was available from earlier investigations [21], and was synthesized according to the method of Bernthsen [28] by heating diphenyl amine (13 mmol), 1naphthoic acid (19.2 mmol) and zinc chloride (29 mmol) for 6 hours at $215 \mathrm{C}$ to give 9-(1-naphthyl)acridine in $50 \%$ yield ( $\mathrm{mp} 232 \mathrm{C}$ ). This was quaternized with iodomethane followed by ion exchange to give 2 in $79 \%$ yield after purification by repeated recrystallization from methanol/ether. ${ }^{1} \mathrm{H}-\mathrm{NMR}\left(200 \mathrm{MHz} ; \mathrm{CD}_{3} \mathrm{OD}\right)$ : $\delta 5.05$ (s, 3H, $\left.\mathrm{N}^{+}-\mathrm{CH}_{3}\right), 7.00(\mathrm{~d}, 1 \mathrm{H}$, naphthyl), 7.33 (t, 1H, naphthyl), 7.59 (t, 1H, naphthyl), 7.65 (d, 1H, naphthyl), 7.7-7.9 (m, 5H, acridinium-H2,7; acridiniumH1,8; naphthyl-H), 8.14 (d, 1H, naphthyl), 8.30 (d, $1 \mathrm{H}$, naphthyl), 8.42 (t, 2H, acridinium-H3,6), $8.84(\mathrm{~d}, 2 \mathrm{H}$, acridinium-H4,5).

4.2. Instrumentation. Continuous electronic absorption and emission spectra were measured on a Hewlett-Packard 8543 diode spectrophotometer and a SPEX Fluorolog III spectrofluorimeter with a GaAs photomultiplier. The fluorescence lifetime of 2 was determined using a Hamamatsu C5680-21 streak camera coupled to a Chromex IS250 spectrograph for detection and a Coherent Infinity-XPO laser for excitation at $450 \mathrm{~nm}$ (fwhm $2.7 \mathrm{~ns}$ ). The same laser was also used as the excitation source in the nanosecond transient absorption measurements using a set-up slightly modified with respect to that described earlier [29]. As before a small microsecond flash lamp (EG \& G, FX504) was used as the source of white probe light and spectrally resolved detection was done with a Princeton Instruments gated (minimum gatewidth $5 \mathrm{~ns}$ ) intensified CCD camera (ICCD-576-G/RB-EM) coupled to a spectrograph. While alternating shots with laser-on and laseroff as usual provided the I and $\mathrm{I}_{0}$ signals, an additional beam splitter was now placed in the probe light beam before it enters the sample to generate on a separate stripe of the detection CCD a reference spectrum of the white light for every shot, which allows for better compensation in fluctuations of the spectral output of the probe flash.

For femtosecond transient absorption measurements a set-up was used nearly identical to one described before [30]. Excitation pulses (fwhm $130 \mathrm{fs}$, rep. rate $1 \mathrm{kHz}$ ) were derived from a Spectra-Physics Hurricane Titanium:Sapphire regenerative amplifier system via an optical parametric amplifier (Spectra Physics OPA 800). White probe light was generated using the residual fundamental light of the OPA focused on sapphire. The probe light was delayed using a motorized delay line (Physik Instrumente M-531DD). The excitation and probe beams pass through the stirred sample cell (pathlength $2 \mathrm{~mm}$ ) at an angle of ca. 8 degrees. The probe beam emerging from the sample is fiber coupled into a CCD spectrometer (Ocean Optics, PC 2000). A chopper placed in the excitation beam provided I and $\mathrm{I}_{0}$ depending on the status of the chopper (open or closed). Experiments were performed under magic angle condition to circumvent the possible influence on the transient absorption of the solute molecule.

\section{REFERENCES}

[1] M. R. Wasielewski, Chem. Rev. 92 (1992), 435.

[2] D. Gust, T. A. Moore, A. L. Moore, A. N. Macpherson, A. Lopez, J. M. DeGraziano, I. Gouni, E. Bittersman, G. R. Seely, F. Gao, R. A. Nieman, X. C. Ma, L. Demanche, D. K. Luttrell, S. J. Lee, and P. K. Perrigan, J. Am. Chem. Soc. 115 (1993), 11141. 
[3] K. Maruyama, A. Osuka, and N. Mataga, Pure Appl. Chem. 66 (1994), 867.

[4] A. Harriman and J. P. Sauvage, Chem. Soc. Rev. 25 (1996), 41.

[5] Y. Sakata, H. Imahori, H. Tsue, S. Higashida, T. Akiyama, E. Yoshizawa, M. Aoki, K. Yamada, K. Hagiwara, S. Taniguchi, and T. Okada, Pure Appl. Chem. 69 (1997), 1951.

[6] D. Gust, T. A. Moore, and A. L. Moore, Acc. Chem. Res. 34 (2001), 40.

[7] H. Imahori, D. M. Guldi, K. Tamaki, Y. Yoshida, C. Luo, Y. Sakata, and S. Fukuzumi, J. Am. Chem. Soc. 123 (2001), 6617.

[8] G. Kodis, P. A. Liddell, L. de la Garza, S. Lin, A. L. Moore, T. A. Moore, and D. Gust, J. Phys. Chem. A 106 (2002), 2036.

[9] K. J. Smit and J. M. Warman, J. Lumin. 42 (1988), 149.

[10] D. Anglos, V. Bindra, and A. Kuki, J. Chem. Soc., Chem. Commun. 2 (1994), 213.

[11] L. Hviid, A. M. Brouwer, M. N. Paddon-Row, and J. W. Verhoeven, Chem. Phys. Chem. 2 (2001), 232.

[12] J. W. Verhoeven, Intern. J. Photoenergy 3 (2001), 79.

[13] S. Fukuzumi, H. Kotani, K. Ohkubo, S. Ogo, N. V. Tkachenko, and H. Lemmetyinen, J. Am. Chem. Soc. 126 (2004), 1600.

[14] T. Hasobe, S. Hattori, H. Kotani, K. Ohkubo, K. Hosomizu, H. Imahori, P. V. Kamat, and S. Fukuzumi, Org. Lett. 6 (2004), 3103.

[15] H. Kotani, K. Ohkubo, and S. Fukuzumi, J. Am. Chem. Soc. 126 (2004), 15999.

[16] A. C. Benniston, A. Harriman, P. Li, J. P. Rostron, and J. W. Verhoeven, Chem. Commun. (2005), submitted.
[17] K. Kasama, K. Kikuchi, Y. Nishida, and H. Kokubun, J. Phys. Chem. 85 (1981), 4148.

[18] K. Kikuchi, C. Sato, M. Watabe, H. Ikeda, Y. Takahashi, and T. Miyashi, J. Am. Chem. Soc. 115 (1993), 5180.

[19] Y. Nishida, K. Kikuchi, and H. Kokubun, J. Photochem. 13 (1980), 75.

[20] S. A. Jonker, F. Ariese, and J. W. Verhoeven, Recl. Trav. Chim. Pays-Bas 108 (1989), 109.

[21] S. A. Jonker, PhD thesis, University of Amsterdam (Amsterdam), 1989.

[22] G. Jones II, M. S. Farahat, S. R. Greenfield, D. J. Gosztola, and M. R. Wasielewski, Chem. Phys. Lett. 229 (1994), 40.

[23] H. van Willigen, G. Jones II, and M. S. Farahat, J. Phys. Chem. 100 (1996), 3312.

[24] N. W. Koper, S. A. Jonker, and J. W. Verhoeven, Recl. Trav. Chim. Pays-Bas 104 (1985), 296.

[25] T. Shida, Electronic Absorption Spectra of Radical Ions, Elsevier, Amsterdam, 1988.

[26] S. Fukuzumi, K. Ohkubo, T. Suenobo, K. Kato, M. Fujitsuka, and O. Ito, J. Am. Chem. Soc. 123 (2001), 8459.

[27] M. E. Peover and B. S. White, J. Electroanal. Chem. 13 (1967), 93.

[28] A. Bernthsen, Liebigs Ann. Chem. 224 (1884), 1.

[29] W. D. Oosterbaan, M. Koeberg, J. Piris, R. W. A. Havenith, C. A. van Walree, B. R. Wegewijs, L. W. Jenneskens, and J. W. Verhoeven, J. Phys. Chem. A 105 (2001), 5984.

[30] F. W. Vergeer, C. J. Kleverlaan, and D. J. Stufkens, Inorg. Chim. Acta 327 (2002), 126. 


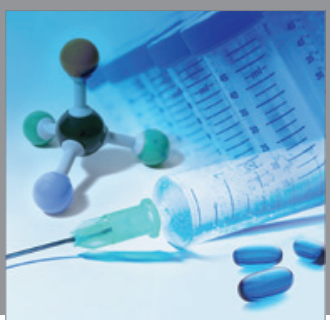

International Journal of

Medicinal Chemistry

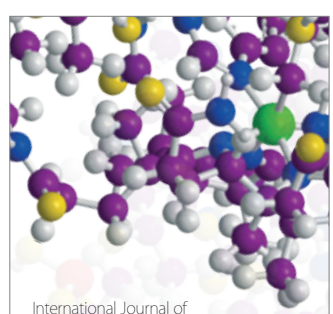

Carbohydrate Chemistry

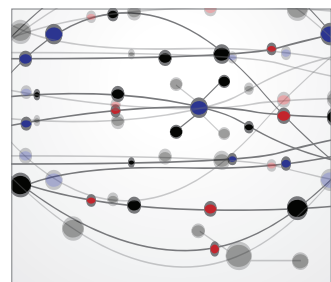

The Scientific World Journal
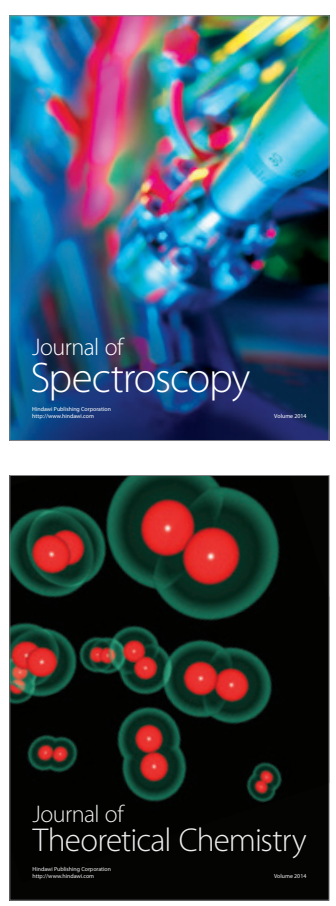
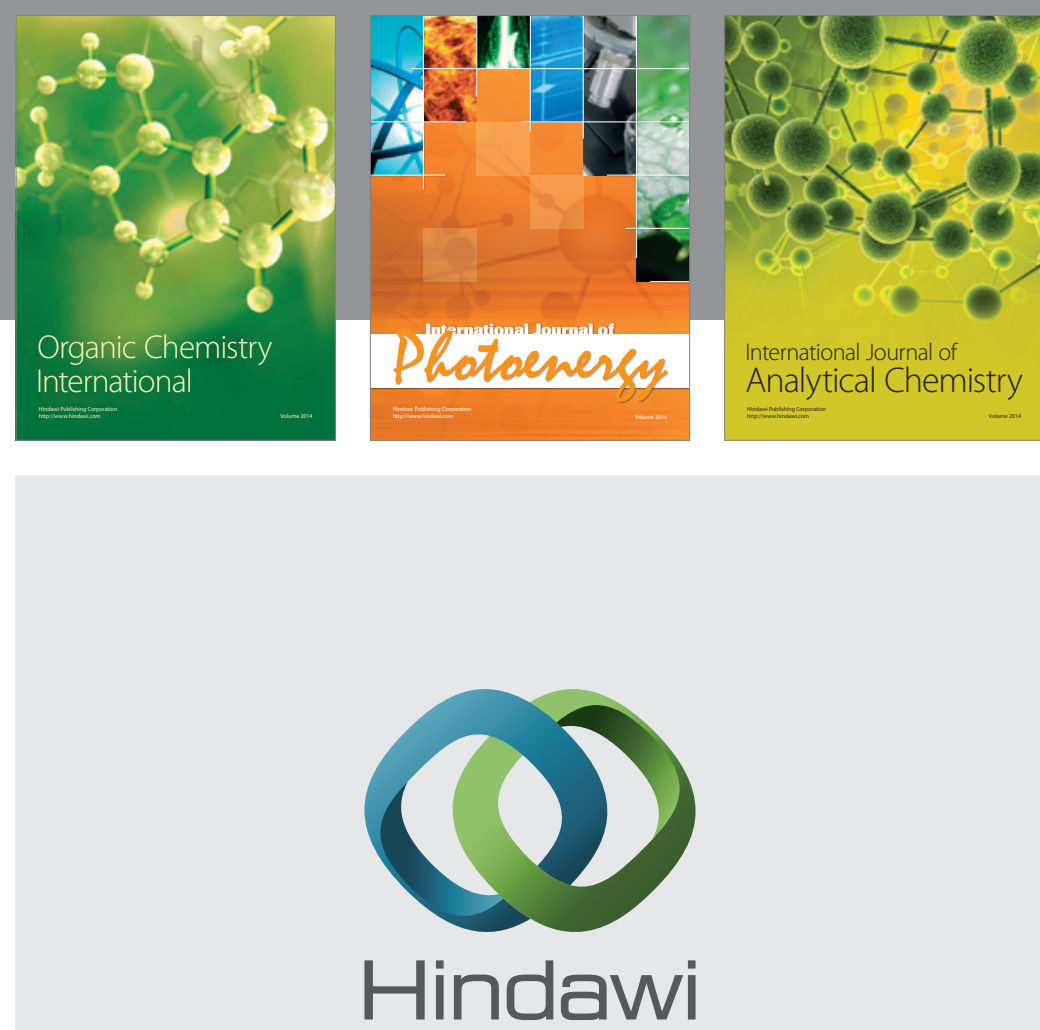

Submit your manuscripts at

http://www.hindawi.com
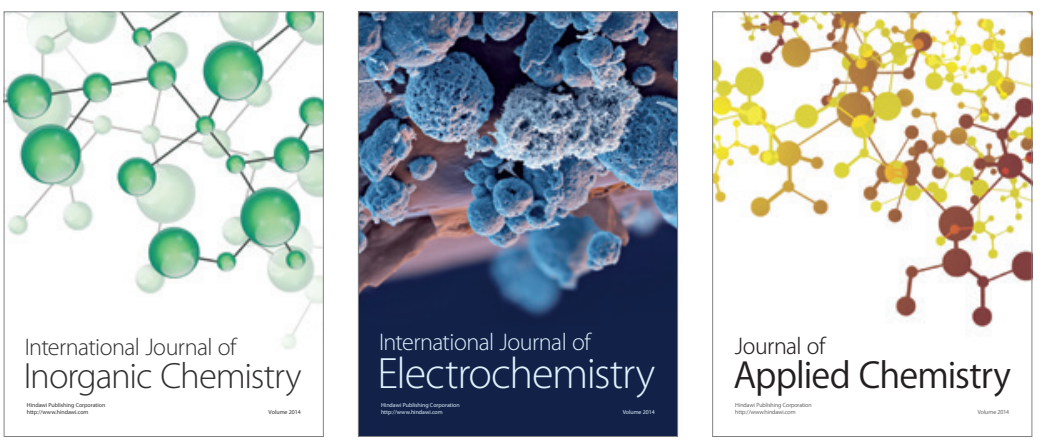

Journal of

Applied Chemistry
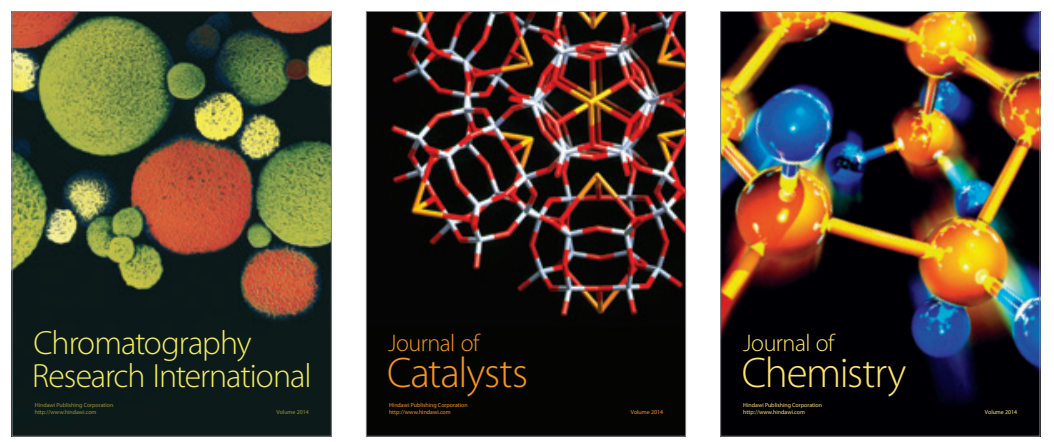
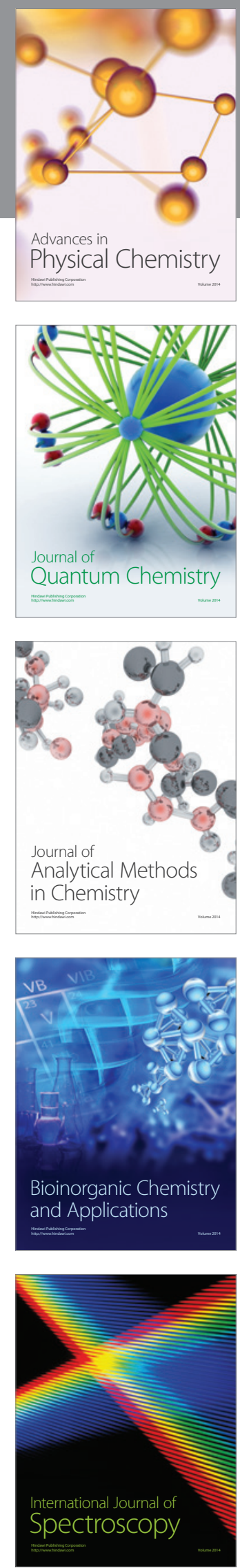